\title{
Visceral Ischemia During Type B Dissection: Incidence, Prognostic Value, Diagnosis, and Operative Management
}

\author{
Domenico Spinelli, Hector W. L. de Beaufort, \\ and Santi Trimarchi
}

\subsection{Introduction}

Organ malperfusion is a possible complication of acute Type B aortic dissection (AD). Theoretically each branch arising from dissected aorta or downstream may be affected, resulting in end-organ ischemia. Obstruction of the celiac and/or mesenteric arteries may lead to visceral ischemia, which is one of the most lethal complications of $\mathrm{AD}$. A timely diagnosis and treatment are crucial to increase the chances of survival. Nowadays, the endovascular approach is the most frequently adopted in order to restore blood flow to the bowel, while open surgery is usually reserved for the cases of failure of endovascular treatment. In this chapter, the incidence, prognostic value, diagnosis, and operative manage-

D. Spinelli

Thoracic Aortic Research Center, IRCCS Policlinico San Donato, San Donato Milanese, Italy

Department of Biomedical and Dental Sciences and Morphofunctional Imaging, Policlinico G. Martino, University of Messina, Messina, Italy

H. W. L. de Beaufort

Thoracic Aortic Research Center, IRCCS Policlinico San Donato, San Donato Milanese, Italy

S. Trimarchi $(\bowtie)$

Thoracic Aortic Research Center, IRCCS Policlinico San Donato, San Donato Milanese, Italy

Department of Scienze Biomediche per la Salute, University of Milan, Milan, Italy

e-mail: santi.trimarchi@unimi.it ment of this dreaded complication of $\mathrm{AD}$ will be described.

\subsection{Incidence and Prognostic Value}

Two different mechanisms can lead to branch vessel obstruction: dynamic obstruction and static obstruction. The most common, occurring in about $80 \%$ of cases, is the dynamic obstruction. The incidence of malperfusion syndrome in acute Type B AD is about $20 \%$ [1], with mesenteric ischemia accounting for about one third of cases. Among complicated ATBAD, contemporary series report an incidence of visceral ischemia ranging from 14 to $25 \%$ [2]. Overall, in-hospital mortality directly related to the visceral ischemia ranges from 2 to $11 \%$ [2]. In the IRAD registry, visceral ischemia of TBAD patients was $7 \%$, and it heavily affected in-hospital mortality, which was more than triplicated compared to patients without visceral ischemia (31\% versus $9 \%$ ) [3]. In this cohort, patients with visceral ischemia were slightly but significantly younger than patients without visceral ischemia (59 vs 64 years) and often had malperfusion of other organs associated. On average, Type B AD patients with visceral ischemia are younger than patients without visceral ischemia [3, 4]. 


\subsection{Diagnosis}

In $\mathrm{AD}$, malperfusion syndrome may become evident through onset of end-organ specific signs of ischemia. It is usually more severe in case of static malperfusion, while it may be intermittent and of varying intensity in case of dynamic obstruction. In this case, care must be taken not to overlook the signs of ischemia, which can lead to a delay in diagnosis. Abdominal pain is present in the majority of cases of visceral ischemia. Nonetheless it is not an exclusive sign of malperfusion syndrome in $\mathrm{AD}$, as it was reported to be present in $40 \%$ of patients without visceral ischemia [3]. Vomiting, diarrhea, and bloody defecation are more specific but later signs. Malperfusion of visceral organs may be associated with malperfusion of other districts. Kidney malperfusion with acute renal failure was reported in $40 \%$ of cases with celiac-mesenteric ischemia and only in $14 \%$ without. Femoral pulse deficit may be present, and a $28 \%$ rate of lower limb ischemia has been reported in patients with visceral malperfusion, while in patients without visceral malperfusion, it was $13 \%$. Also spinal cord ischemia has been reported more often in association with visceral malperfusion (5\% versus $2 \%$ ). The suspicion of visceral malperfusion should increase in case of positivization of blood markers of ischemia. Levels of D-dimer, lactate, ammonia, transaminase, creatine phosphokinase, and leukocytes may be elevated. Recently, other markers of mesenteric ischemia have been tested. The intestinal fatty acid-binding protein (I-FABP) was reported to have a sensitivity of $79 \%$ and a specificity of $91 \%$ [5]. However many markers of ischemia increase only after the intestine has become necrotic. Therefore, negative biochemical markers should not delay intervention if there is a strong suspicion of visceral malperfusion. In presence of clear peritoneal signs, the patient should be treated without hesitation. Imaging evidence of visceral malperfusion syndrome includes true lumen compression, branch vessel dissection and thrombosis, and reduced late-phase visceral enhancement on computed tomography angiography (CTA) (Fig. 29.1). Doppler ultrasonography can show arterial thrombosis, dissection, or patterns of altered flow, but the sensitivity is not high.

\subsection{Operative Management}

Thoracic endovascular aortic repair (TEVAR) is currently the first-line treatment in acute complicated Type B AD patients [6, 7]. The treatment consists in the coverage of the proximal entry tear in order to achieve expansion of the true lumen and decompression of the false lumen. Thus, celiac or mesenteric artery obstruction are reversed in most cases. Otherwise, additional branch vessel stenting may be necessary in up to $20 \%$ of the cases [8-10]. TEVAR is therefore effective in treating mesenteric malperfusion in the setting of dynamic obstruction [8]. However, in-hospital mortality rates are still considerable, ranging from 20 to $40 \%$ [9-11].

Furthermore, TEVAR-related complications may also occur in a small percentage of cases. Retrograde Type A AD and stent graft-induced new entry tears have been described, in particular in relation to the use of stent grafts provided of proximal bare spring $[12,13]$ or in case of excessive oversizing [14]. In order to prevent such complications, the stent graft oversizing in Type B AD should not exceed $10 \%$, and the proximal edge of the stent graft should be deployed in a non-dissected zone. The use of a distal aortic bare metal stent in combination with the standard TEVAR (PETTICOAT-Provisional Extension To Induce Complete ATtachment-technique) [15] has been proposed both for the treatment of persistence of malperfusion after TEVAR and for the prevention of late complications. In the long run, complications may occur in patients treated with TEVAR, and in particular an aneurysmal evolution should be expected. In these cases, an endovascular treatment with branched stent graft is feasible, but additional difficulties should be anticipated, especially if the visceral vessels originate from different lumina $[16,17]$.

Endovascular fenestration of the dissection flap is a valid treatment option in case of persistence of false lumen pressurization and dynamic obstruction. It can be performed also as 

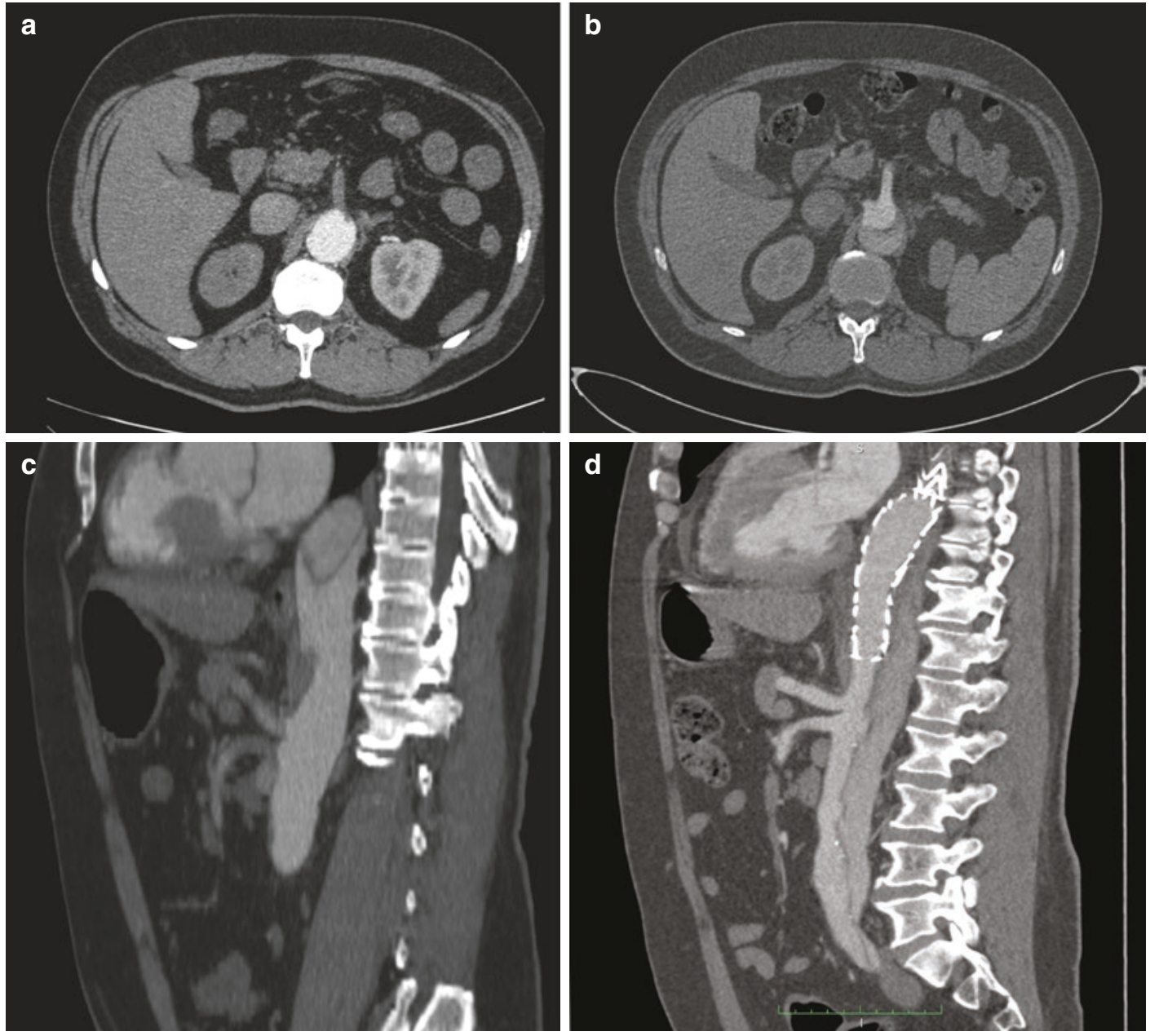

Fig. 29.1 Dynamic obstruction of visceral arteries (a axial view, c sagittal view), resolved after TEVAR (b axial view, d sagittal view)

an alternative to TEVAR. If necessary, it can be accompanied by branch vessel stenting if static obstruction of a branch vessel is present.

A $17 \%$ early mortality rate was reported after endovascular fenestration in a cohort of 69 Type $\mathrm{B}$ AD patients with malperfusion performed in an experienced center. Mortality was due to aortic rupture in $7 \%$ of cases and malperfusion complications in $10 \%$ [18]. Following endovascular fenestration, even more than after TEVAR, the risk of late aortic dilatation should be taken into account. Freedom from aortic rupture or aortic repair rates at 1,5 , and 8 years of $80 \%, 68 \%$, and $54 \%$ have been reported [18]. The technique of endovascular flap fenestration entails gaining access to both lumina as a first step. In this phase, intravascular or intraesophageal ultrasonography may be of great help to distinguish the two lumina and identify the tears. It also helps reduce the use of iodinated contrast medium, which is particularly important in case of renal malperfusion to lower the risk of renal failure [15]. Pressure measurements allow to detect a pressure gradient between the two lumina, as well as between the proximal aorta and the branch vessels, providing an objective assessment of malperfusion. It is important to document the patency status of all branches before and after the procedure, as the resolution of obstruction in one branch vessel may lead to unpredictable changes in mobil- 
ity of the dissection flap and potentially cause dynamic obstruction of other branch vessels. If static obstruction of a branch vessel is found, this should be treated by stenting prior to the fenestration procedure. In order to perform fenestration, the dissection flap needs to be crossed through a reentry tear, which is usually present at the level of branch vessels. Because the true lumen is smaller, it is usually easier to cross from the true lumen to the false lumen. If no reentry tears are found, it is possible to pierce the dissection flap using a catheter and a stiff guidewire at the level of the obstructed artery. Then a guidewire is advanced through the fenestration, and serial balloon angioplasty is performed until the pressure differential between the two lumina is abolished. If necessary, a bare metal stent may be placed in the true lumen to preserve patency of the fenestration. Other fenestration techniques have been described, such as the scissor technique and the cheese wire fenestration technique. In the latter, the guidewire, after crossing the septum, is captured with a snare device and pulled out through the contralateral groin access. Then the guidewire is pulled inferiorly with a sawing motion to shear the dissection flap, stopping before the aortic bifurcation. However, pulling the flap downward carries a risk of circumferential detachment of the intimal layer, which can result in aortic intussusception and obstruction [15]. TEVAR is currently considered the first-line treatment in most cases [7]. Fenestration may be more appropriate than TEVAR in patients with increased risk of spinal cord ischemia such as those with history of previous abdominal aortic repair and occlusion of hypogastric, subclavian, or vertebral artery. On the contrary, TEVAR is particularly indicated in elderly patients, in case of aortic dilatation, and for intermediate-risk patients (i.e., patients with refractory/recurrent pain or hypertension). Overall, the endovascular approach was used in almost $70 \%$ of patients with visceral ischemia enrolled in the IRAD in recent years [3]. In case of suspicion of intestinal infarction, an explorative laparoscopy or laparotomy is indicated. Acute abdomen, refractory lactic acidosis, and hemodynamic instability due to ongoing intestinal malperfusion warrant immediate laparotomy and resection of necrotic intestinal tracts. A temporary closure technique such as Bogota bag or VAC closure system can be used to allow later inspection of the abdominal organs. In case endovascular techniques fail to restore vascularization to the visceral arteries, open surgery is necessary [3]. Surgical vascular reconstruction should also be considered in case of anatomical contraindication for TEVAR, such as unsuitable iliac accesses and insufficient proximal landing zones [6]. Furthermore, for patients with connective tissue disorders, TEVAR can be used as a bridge treatment, while open surgical aortic replacement is the treatment of choice. Open surgery should be considered also in younger patients, since longterm durability of TEVAR is still unclear.

Open surgical options to treat visceral malperfusion include open fenestration and aortic graft replacement. Open fenestration requires exposure of the visceral segment of the aorta and clamping in supraceliac position. A longitudinal aortotomy is then carried out on the left anterolateral aortic wall, and the dissection flap is widely resected to maximize the size of the single aortic lumen. The restoration of flow in the branch vessels may be assessed through intraoperative Doppler ultrasound.

Although open surgical fenestration appears to be effective at relieving malperfusion, it is still burdened by rates of in-hospital mortality of $20 \%$ [19]. There are concerns about late aneurysmal evolution after surgical fenestration. However positive results have been reported in this regard when reduction of aortic diameter at the closure of aortotomy was carried out. In fact, according to the law of Laplace, the tension on the aortic wall decreases by reducing the radius [19].

Graft replacement of thoracic or thoracoabdominal aorta is a more definitive option [3]. However, in the acute setting, in AD patients, it is associated with about $30 \%$ mortality [20] and a relevant risk of spinal cord ischemia in case of extensive distal thoracoabdominal resection [3]. Although the visceral malperfusion is an absolute indication for invasive treatment, the rate of patients with visceral ischemia who only receive medical management is around $20 \%$ in IRAD [3]. 
The reason for this discrepancy may be partially due to the presence in multicenter registries of patients with radiological or angiographic signs suggestive of mesenteric obstruction without matching clinical signs [21]. More often, these patients are in such a poor clinical condition that are deemed unsuitable for endovascular or surgical intervention, and an attempt of operative management is denied. In this class of patients, the mortality rate reaches $50 \%$. Indeed, in these patients the conservative treatment independently predicted in-hospital mortality [3]. Conversely, mortality rates after surgical and endovascular management of visceral ischemia were comparable $(25.8 \%$ and $25.5 \%)$. However, the endovascular approach is considered the firstline treatment $[6,7]$.

\subsection{Conclusion}

Visceral ischemia is a life-threatening complication of acute Type B AD that mandates prompt intervention. Timeliness of diagnosis and treatment is crucial in order to successfully reverse end-organ ischemia. TEVAR is the first-line treatment. Endovascular fenestration can be used either as an alternative to TEVAR in selected patients or after TEVAR when it was not effective in restoring perfusion to the affected branch vessels. Open surgery should be limited to cases in which endovascular techniques are contraindicated or unsuccessful.

\section{References}

1. Suzuki T, Mehta RH, Ince H, et al. Clinical profiles and outcomes of acute type B aortic dissection in the current era: lessons from the International Registry of Aortic Dissection (IRAD). Circulation. 2003;108:III312-7.

2. Kamman AV, Yang B, Kim KM, Williams DM, Deeb GM, Patel HJ. Visceral malperfusion in aortic dissection: the Michigan experience. In: Seminars in thoracic and cardiovascular surgery. Philadelphia: W. B. Saunders; 2016.

3. Jonker FH, Patel HJ, Upchurch GR, et al. Acute type $\mathrm{B}$ aortic dissection complicated by visceral ischemia. J Thorac Cardiovasc Surg. 2015;149(4):1081-6.e1.
4. Jonker FH, Trimarchi S, Muhs BE, et al. The role of age in complicated acute type B aortic dissection. Ann Thorac Surg. 2013;96(6):2129-34.

5. Treskes N, Persoon AM, van Zanten AR. Diagnostic accuracy of novel serological biomarkers to detect acute mesenteric ischemia: a systematic review and meta-analysis. Intern Emerg Med. 2017;12(6):1-6.

6. Fattori R, Cao P, De Rango P, et al. Interdisciplinary expert consensus document on management of type B aortic dissection. J Am Coll Cardiol. 2013;61(16):1661-78.

7. Riambau V, Böckler D, Brunkwall J, Cao P, Chiesa R, Coppi G, Czerny M, Fraedrich G, Haulon S, Jacobs MJ, Lachat ML. Editor's choice-management of descending thoracic aorta diseases. Eur $\mathrm{J}$ Vasc Endovasc Surg. 2017;53(1):4-52.

8. Szeto WY, McGarvey M, Pochettino A, et al. Results of a new surgical paradigm: endovascular repair for acute complicated type B aortic dissection. Ann Thorac Surg. 2008;86(1):87-93; discussion 93-4.

9. Sfyroeras GS, Rubio V, Pagan P, Diethrich EB, Rodriguez JA. Endovascular management of malperfusion in acute type B aortic dissections. J Endovasc Ther. 2011;18(1):78-86.

10. Ryan C, Vargas L, Mastracci T, et al. Progress in management of malperfusion syndrome from type $\mathrm{B}$ dissections. J Vasc Surg. 2013;57(5):1283-90; discussion 90 .

11. Khoynezhad A, Donayre CE, Omari BO, Kopchok GE, Walot I, White RA. Midterm results of endovascular treatment of complicated acute type B aortic dissection. J Thorac Cardiovasc Surg. 2009;138(3):625-31.

12. Dong ZH, Fu WG, Wang YQ, da Guo Q, Xu X, Ji Y, et al. Retrograde type A aortic dissection after endovascular stent graft placement for treatment of type B dissection. Circulation. 2009; 119:735-41.

13. Eggebrecht H, Thompson M, Rousseau H, Czerny M, Lönn L, Mehta RH, et al. Retrograde ascending aortic dissection during or after thoracic aortic stent graft placement: insight from the European registry on endovascular aortic repair complications. Circulation. 2009;120(11 Suppl):S276-81.

14. Canaud L, Ozdemir BA, Patterson BO, Holt PJ, Loftus IM, Thompson MM. Retrograde aortic dissection after thoracic endovascular aortic repair. Ann Surg. 2014;260:389-95.

15. Canaud L, Faure EM, Ozdemir BA, Alric P, Thompson M. Systematic review of outcomes of combined proximal stent-grafting with distal bare stenting for management of aortic dissection. Ann Cardiothorac Surg. 2014;3(3):223-33.

16. Oikonomou K, Kopp R, Katsargyris A, Pfister K, Verhoeven EL, Kasprzak P. Outcomes of fenestrated/ branched endografting in post-dissection thoracoabdominal aortic aneurysms. Eur J Vasc Endovasc Surg. 2014;48(6):641-8.

17. Oikonomou K, Katsargyris A, Ritter W, Spinelli D, Seto Y, Verhoeven EL. Endovascular management of 
chronic post-dissection aneurysms. Ann Cardiothorac Surg. 2014;3(3):307.

18. Patel HJ, Williams DM, Meerkov M, Dasika NL, Upchurch GR Jr, Deeb GM. Long-term results of percutaneous management of malperfusion in acute type B aortic dissection: implications for thoracic aortic endovascular repair. J Thorac Cardiovasc Surg. 2009;138(2):300-8.

19. Trimarchi S, Segreti S, Grassi V, et al. Open fenestration for complicated acute aortic B dissection. Ann Cardiothorac Surg. 2014;3(4):418-22.
20. Conrad M, Cambria R. Aortic dissection. In: Cronenwett J, Johnston KW, editors. Rutherford's vascular surgery. 8th ed. Philadelphia: Elsevier Saunders; 2014. p. 2179-85.

21. Lauterbach SR, Cambria RP, Brewster DC, et al. Contemporary management of aortic branch compromise resulting from acute aortic dissection. J Vasc Surg. 2001;33(6):1185-92. 